\title{
Uso de ácidos graxos poli-insaturados durante a gestação: Um estudo bibliográfico
}

\author{
Use of polyunsaturated fatty acids during gestation: A bibliographic study
}

Uso de ácidos grasos poliinsaturados durante la gestación: Un estudio bibliográfico

Evelise Staevie dos Santos ${ }^{1 *}$, Danielle Monteiro Fonseca da Silva ${ }^{1}$, Taiane Carvalho Frota ${ }^{1}$, Yris Roxana Garcia Vasquez¹.

\section{RESUMO}

Objetivo: Realizar uma revisão na literatura científica, sobre a ação dos ácidos graxos, em especial ômega3 e ômega- 6 durante o período gestacional. Métodos: Abordagem do tipo revisão bibliográfica. A coleta de dados foi realizada entre os meses de setembro e novembro de 2018 , utilizando como descritores "Fatty acids and pregnancy" "Use of omega in pregnancy", "Mechanism of action of the omega in pregnancy" e "Use of omega in pregnancy, advantages and disadvantages", inseridos nas bases de dados PUBMED, Science Direct, NCBI e Scielo, usando como critério de inclusão artigos originais disponíveis, publicados no período de 2010 a 2018, nos idiomas inglês e português. Resultados: Dos 19 artigos que compõem essa pesquisa, constatou-se que o uso de ômega, principalmente da classe 3 são necessários para o período gestacional, por contribuírem para o bem-estar da gestante e do concepto, prevenindo parto prematuro, pré eclâmpsia, baixo peso do bebê, dentre outros. Conclusão: Com base nas pesquisas recentes a respeito do uso de ácidos graxos poli-insaturados, foi possível constatar que a suplementação com ômega-3 é favorável ao binômio materno-fetal.

Palavras-chave: Ômega-3, Ômega-6, Gestação.

\begin{abstract}
To perform a review in the scientific literature on the action of fatty acids, especially omega- 3 and omega- 6 during the gestational period. Methods: Bibliographic review approach. Data collection was performed between September and November 2018, using as descriptors "Fatty acids and pregnancy" "Use of omega in pregnancy", "Mechanism of action of the omega in pregnancy" and "Use of omega in pregnancy, advantages and disadvantages", inserted in the databases PUBMED, Science Direct, NCBI and Scielo, using as inclusion criterion original articles available, published between 2010 and 2018, in the English and Portuguese languages. Results: Of the 19 articles that compose this research, it was verified that the use of omega, mainly of class 3 are necessary for the gestational period, because they contribute to the well-being of the pregnant woman and the concept, preventing premature birth, preeclampsia, low weight of the baby, and others. Conclusion: Based on recent research on the use of polyunsaturated fatty acids, it was possible to verify that the supplementation with omega- 3 is favorable for the maternal-fetal binomial.
\end{abstract}

Keywords: Omega 3, Omega-6, Gestation.

${ }^{1}$ Universidade Federal do Amazonas (UFAM), Manaus-Amazonas. *E-mail: evelise s@hotmail.com 


\section{RESUMEN}

Realizo una revisión en la literatura científica, sobre la acción de los ácidos grasos, especialmente omega-3 y omega-6 durante el período gestacional. Métodos: Enfoque del tipo revisión bibliográfica. La colecta de datos fuerón realizadas entre setiembre y noviembre de 2018, utilizando como descriptores "Fatty acids and pregnancy", "Use of omega in pregnancy", "Mechanism of action of the omega in pregnancy" y "Use of omega in pregnancy, advantages and disadvantages" insertado em las bases de dados PubMed, Science Direct, NCBI y Scielo, usando como criterio de inclusión artículos originales disponibles y publicados en el período 2010 hasta 2018, em lós idiomas Inglés y portugués. Resultados: De los 19 artículos que componen esta búsqueta, se constató que el uso de omega, principalmente de la clase 3 son necesarios para el período gestacional, por contribuir em el bienestar de la gestante y del concepto, previniendo parto prematuro, pre-eclampsia, bajo peso del bebé, entre otros. Conclusión: Con base en la búsqueta recientes al respecto del uso de ácidos grasos poliinsaturados, fue posible constatar que la suplementación con omega-3 es favorable para el binomio materno-fetal.

Palabras clave: Omega 3, Omega 6, Gestación.

\section{INTRODUÇÃO}

A gestação é caracterizada como um momento de mudanças para a mulher, com alterações fisiológicas, metabólicas, físicas e psicológicas, onde o cuidado com saúde da mãe e do feto que está sendo gerado necessita de extrema atenção. Tal período está associado a fatores ambientais que podem influenciar a formação do feto, dentre eles, a alimentação inadequada durante o período de gestação, consumo de gordura e óleos com alta proporção de ômega 6 e baixa proporção de ômega 3, o que é muito comum em gestações frequentes e múltiplas, por diminuir as reservas desses ácidos graxos de cadeia longa. Sendo então recomendado ganho de peso, consumo de alimentos com variedade e quantidade determinadas para suprir as necessidades energéticas e nutricionais de ambos (MOURA e AZEVEDO, 2018) (MEREY et al., 2017).

Nos dias atuais, hábitos alimentares saudáveis são preconizados, optando - se por alimentos com elevados índices nutricionais e redução de gorduras, dando preferências àquelas compostas por ácidos graxos, especialmente no período gestacional, onde o transporte de alimentos é realizado por via placentária e depositados, como no caso dos ácidos graxos do tipo ômega, no cérebro e retina do feto (CAMPOS et al., 2013).

Mudanças na nutrição materna podem ter influências diretas no seguimento da gestação, principalmente com relação à duração e ao crescimento do feto, dentre esses, os ácidos graxos poli-insaturados de cadeia longa das séries ômega 3 (n-3), como o ácido linolênico, e ômega 6 (n-6), como o ácido linoléico, destacam-se quanto a benefícios à saúde materna e da criança, apresentando ação no desenvolvimento cerebral e na retina do feto, menor incidência de alergias e doenças asmáticas em crianças durante a infância e prevenção de doenças crônicas (GOULD et al., 2016) (GUNARTE et al, 2015) (INNIS, 2014) (ZHAO et al, 2014).

Ácidos graxos (AG) poli-insaturados são caracterizados quimicamente como uma cadeia longa, de 14 a 22 átomos de carbono. São representantes desses grupos o ácido docosahexaenóico (DHA), ácido eicosapentaenóico (EPA) e ácido araquidônico (AA), encontrados em alimentos de origem animal e vegetal (OLIVEIRA, LUZIA E RONDÓ, 2015).

A recomendação de ingestão de lipídeos totais e ácidos graxos para a gestante assemelha-se ao sugerido a população geral, com a indicação de maior consumo de ômega 3. De acordo com Koletzko et al (2007), é recomendado durante a gestação, o consumo mínimo de $200 \mathrm{mg} /$ dia de DHA, quantidade a qual 
pode ser adquirida em uma ou duas porções por semana de peixes marinhos gordurosos, tais como arenque, cavala e principalmente, sardinha e salmão. Já Fao (2010), recomenda durante o período gestacional e de lactação, o consumo mínimo de $200 \mathrm{mg} /$ dia de DHA e $300 \mathrm{mg} /$ dia de DHA associado a EPA. Até o presente momento, não foram estabelecidas exigências quantitativas de valores quanto a ingestão de ácidos graxos do tipo ômega, principalmente ômega 3 e ômega 6 , em gestantes, sendo admitido a variação entre 5:1 a 10:1 (SIMOPOULOS, 2002).

Deve-se promover a ingestão de tais ácidos graxos do tipo ômega principalmente devido ao consumo inadequado de alimentos que servem como fonte de tais nutrientes na dieta, com a utilização suplementos alimentares, por exemplo, com óleos de peixes e/ou derivados, porém, o alto custo de tais complementos inviabiliza a utilização por gestantes de classes econômica menos favorecida (OLIVEIRA et al., 2015) (BOKOR et al., 2010).

De forma geral, elevadas concentrações de ácidos graxos poli-insaturados da série ômega 3 na gestação, principalmente (DHA), apresentam melhores resultados na gestação, promovendo tempo ideal de gestação, ajudando a evitar partos prematuros. Em contrapartida, os da série ômega 6 podem influenciar de forma negativa o desfecho gestacional, influenciando diretamente na ação de prostaglandinas, e assim, incitando eventos adversos como exemplo um parto prematuro (BEST et al, 2016) (KAR et al, 2015) (GUNARTE et al, 2015) (INNIS, 2014).

Com base no que foi exposto, o objetivo desse trabalho foi realizar um levantamento bibliográfico sobre as principais informações relacionadas a utilização de ácidos graxos do tipo ômega-3 e ômega- 6 durante a gestação, assim como os possíveis mecanismo de ação durante o metabolismo, doses recomendadas e os benefícios ao organismo, tanto da mãe quanto do feto em desenvolvimento.

\section{MÉTODOS}

Como ação metodológica foi utilizado o método de revisão bibliográfica, incluídos estudos com métodos de pesquisas diversificados, organizados sobre o tema, auxiliando a conclusões sobre o assunto.

O trabalho seguiu as etapas: 1) estabelecer a questão de pesquisa; 2) definir critérios de inclusão e exclusão na literatura; 3) classificar os estudos; 4) avaliar os estudos incluídos na revisão; 5) interpretar os resultados; 6) sintetizar o conhecimento (MENDES, SILVEIRA E GALVÃO, 2008).

A pesquisa em banco de dados foi realizada entre os meses de setembro - novembro de 2018, nas bases PUBMED, Science Direct, NCBI e Scielo. Foram determinados como critérios de inclusão artigos originais disponíveis, publicados no período de 2010 a 2018, nos idiomas inglês e português. Para elaboração da questão norteadora, a técnica $\mathrm{PVO}$, onde $\mathrm{P}$ refere-se ao problema, os participantes ou o contexto inserido; $V$ corresponde as variáveis do estudo; $\mathrm{O}$ compreende o desfecho ou resultado esperado. Com essa base, foi elaborada a questão: Qual a ação de ácidos graxos poli-insaturados durante o período gestacional?

Os artigos foram organizados de acordo com variáveis e bases de dados, determinando como problema $(\mathrm{P})$ o descritor "Omega in pregnancy", como variável (V) utilizou-se "Fatty acids and pregnancy" "Use of omega in pregnancy", "Mechanism of action of the omega in pregnancy" e no Outcome $(O)$ foi utilizado "Use of omega in pregnancy, advantages and disadvantages". Após realização da busca em base de dados citadas, foram encontrados 247 artigos científicos, dentre os quais, 200 na PUBMED, 37 na Science Direct, 4 no NCBI e 6 na Scielo. Destes, foi feita a análise de duplicatas e adequação aos critérios de inclusão, obtendo-se após exclusão 110 artigos para avaliação de conteúdo, que após esta avaliação, se enquadraram com o objetivo da pesquisa 19 artigos, os quais compuseram a pesquisa (Figura 1). 
Figura 1. Fluxograma de seleção dos artigos que integram a pesquisa.

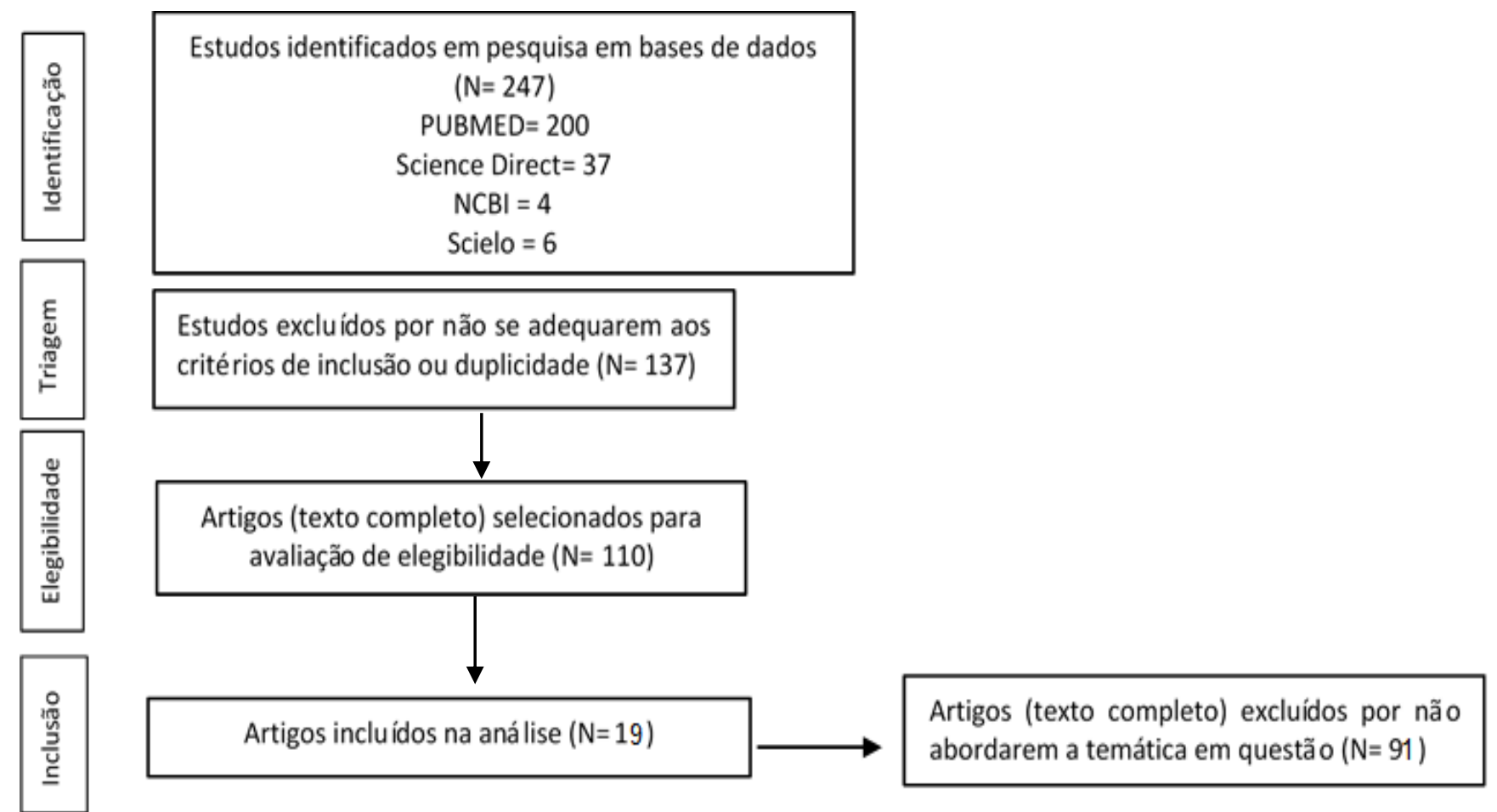

Fonte: STAEVIE, 2018.

\section{RESULTADOS}

Foram utilizados artigos que discorriam questões sobre a ação de ácidos graxo do tipo ômega 3 e ômega 6 durante o período gestacional, informando: 1) o valor de doses recomendadas; 2) benefícios do uso do ômega para mãe e feto 3) mecanismo de ação e absorção. Para uma melhor compreensão e entendimento, os estudos foram organizados por descrição de algumas características, como: autores, ano e país de publicação, periódico, objetivos, interpretação do estudo e resultados.

Com relação ao ano de publicação, como apresentado no Quadro 1, foram apresentados 19 artigos, 02 trabalhos em 2010 e 2013, 04 trabalhos entre 2014 e 2015, 05 trabalhos em 2016, 03 trabalhos em 2017 e maior prevalência de estudos no ano de 2018, com 05 trabalhos publicados. Quanto ao país de publicação, destaca - se o Brasil, seguido por Reino Unido, Canadá, Irã, Índia, China, Itália e México, respectivamente. Com relação ao tipo de estudo, todos os artigos aqui utilizados são oriundos de pesquisas originais, realizadas diretamente com pacientes ou banco de dados, abrangendo estudos experimentais, retrospectivos, quantitativos, descritivos e de meta-análise. Os periódicos são nacionais e internacionais, de áreas médicas e nutricionais. 
Quadro 1. Caracterização de artigos incluídos na pesquisa. Manaus, Brasil, 2018.

\begin{tabular}{|c|c|c|c|c|}
\hline Autor/ Ano/ País & $\begin{array}{c}\text { Base de } \\
\text { dado }\end{array}$ & $\begin{array}{l}\text { Tipo de } \\
\text { estudo }\end{array}$ & Objetivo & Interpretação do Estudo \\
\hline $\begin{array}{l}\text { GRIGORIADIS } \\
\text { et al } \\
\text { Canadá/2010 }\end{array}$ & $\begin{array}{l}\text { Science } \\
\text { Direct }\end{array}$ & $\begin{array}{l}\text { Prospectiva } \\
\text { longitudinal }\end{array}$ & $\begin{array}{l}\text { Analisar a frequência do uso ômega-3, } \\
\text { multivitamínico e outros suplementos em } \\
\text { mulheres grávidas }\end{array}$ & $\begin{array}{l}\text { Estudo com } 176 \text { mulheres, seis estavam no primeiro trimestre de gravidez, } 82 \\
\text { no segundo trimestre e } 88 \text { no terceiro trimestre. Dentre elas, } 156 \text { utilizavam } \\
\text { multivitamínico suplementados com ômega-3 e } 20 \text { apenas ômega-3. Os } \\
\text { resultados indicam que mulheres grávidas de origem canadense fazem uso } \\
\text { de multivitamínicos ricos em ômega durante o período gestacional, } \\
\text { contribuindo para sua saúde e do feto. }\end{array}$ \\
\hline $\begin{array}{l}\text { BARTOLLOZZO } \\
\text { et al } \\
\text { Brasil/ } 2013\end{array}$ & Scielo & Experimental & $\begin{array}{l}\text { Avaliar o impacto da suplementação da } \\
\text { dieta de mulheres durante a gestação e a } \\
\text { lactação com óleo de peixe contendo o } \\
\text { ácido docosahexaenóico (DHA) ômega-3 e } \\
\text { sua influência na composição do leite } \\
\text { humano. }\end{array}$ & $\begin{array}{l}\text { Estudo realizado com } 60 \text { mulheres grávidas, submetidas a suplementação } \\
\text { diária com cápsulas de óleo de peixe, com } 315 \mathrm{mg} \text { de DHA e } 80 \mathrm{mg} \text { de EPA } \\
\text { durante o } 3^{\circ} \text { trimestre da gravidez e nos } 03 \text { primeiros meses pós parto. Os } \\
\text { resultados não apresentaram diferença estatística entre o conteúdo de } \\
\text { gordura dos grupos estudo (cápsulas de óleo de peixe) e controle (cápsulas } \\
\text { contendo amido de milho). No entanto, o leite de mulheres que tomavam óleo } \\
\text { de peixe continha níveis mais elevados de DHA e EPA } 30 \text { e } 60 \text { dias pós- } \\
\text { parto. Estes resultados demonstram que a alta ingestão de ômega-3 pode } \\
\text { influenciar sua concentração no leite humano. }\end{array}$ \\
\hline $\begin{array}{l}\text { FEREIDOONI e } \\
\text { JENABI } \\
\text { Irã/ } 2014\end{array}$ & PUBMED & Experimental & $\begin{array}{l}\text { Investigar a taxa de uso de ômega } 3 \text { em } \\
\text { mulheres grávidas e seu efeito sobre } \\
\text { o resultado da gravidez }\end{array}$ & $\begin{array}{l}\text { O estudo foi realizado por } 530 \text { mulheres na } 16^{\circ} \text { semana de gestação, } \\
\text { divididas em } 3 \text { grupos: } 1 \text { ) consumiam peixe } 1 \text { vez/mês; } 2 \text { ) consumiam peixe } \\
1-3 \text { vezes/ mês; } 3 \text { ) consumiam mais de } 3 \text { vezes. Como resultado foi possível } \\
\text { analisar que o consumo de ômega-3 pode prevenir baixo peso ao nascer, } \\
\text { trabalho de parto prematuro e a pré-eclâmpsia. }\end{array}$ \\
\hline $\begin{array}{l}\text { ESCAMILLA- } \\
\text { NUÑEZ et al } \\
\text { México/ } 2014\end{array}$ & $\mathrm{NCBI}$ & Experimental & $\begin{array}{l}\text { Avaliar o consumo pré-natal de ácidos } \\
\text { graxos ômega-3 pode atuar como adjuvante } \\
\text { no desenvolvimento do sistema imunológico } \\
\text { e afetar a resposta inflamatória de } \\
\text { neonatos. }\end{array}$ & $\begin{array}{l}\text { O estudo foi realizado com } 1.094 \text { mulheres grávidas, submetidas a ingestão } \\
\text { de } 400 \mathrm{mg} / \text { dia de ácido (DHA) ou placebo de } 18 \text { a } 22 \text { semanas de gestação } \\
\text { até o parto. Os resultados um efeito protetor estatisticamente significativo do } \\
\text { tratamento com } \mathrm{DHA} \text {, indicando que a suplementação com o mesmo durante } \\
\text { a gravidez pode diminuir a incidência de sintomas respiratórios em crianças } \\
\text { com história de atopia materna }\end{array}$ \\
\hline $\begin{array}{l}\text { SACCONE et al } \\
\text { Itália/ } 2015\end{array}$ & PUBMED & Meta-análise & $\begin{array}{l}\text { Analisar recomendações baseadas em } \\
\text { evidências da suplementação de ômega-3 } \\
\text { durante a gravidez. }\end{array}$ & $\begin{array}{l}\text { O estudo indica que ômega- } 3 \text { não está associado a prevenção de pré- } \\
\text { eclâmpsia (PE), crescimento intrauterino restrito (RCIU), diabetes gestacional } \\
\text { (DG), depressão pós-parto (DPP). Em mulheres suplementadas com ômeg- } \\
3 \text { houve baixa incidência de hiperbilirrubinemia em recém-nascidos. }\end{array}$ \\
\hline
\end{tabular}

REAS/EJCH | Vol. 11 (1) | e218 | DOI: https://doi.org/10.25248/reas.e218.2019 Página 5 de 10 


\begin{tabular}{|c|c|c|c|c|}
\hline $\begin{array}{c}\text { Yu et al } \\
\text { Cingapura/ } 2015\end{array}$ & PUBMED & Coorte & $\begin{array}{l}\text { Investigar relação entre o status materno de } \\
\text { ácidos graxos poliinsaturados de cadeia } \\
\text { longa (PUFA) e potencial alergia à prole em } \\
\text { um grupo multiétnico asiático. }\end{array}$ & $\begin{array}{l}\text { No estudo, foram utilizadas amostras de plasma materno, entre a } 26^{\mathrm{a}}-28^{\text {a }} \\
\text { semana de gestação para abundância relativa de PUFA. Os descendentes ( } \\
960 \text { ) foram acompanhados de } 03 \text { semanas a } 18 \text { meses de idade, e os } \\
\text { desfechos clínicos de possíveis doenças alérgicas, como rinite e eczema. Os } \\
\text { resultados indicaram níveis de PUFA ômega-3 e ômega- } 6 \text { não foram } \\
\text { significativamente associados à rinite e eczema. }\end{array}$ \\
\hline $\begin{array}{l}\text { AKERELE e } \\
\text { CHEEMA } \\
\text { Canadá/2016 }\end{array}$ & $\begin{array}{c}\text { Science } \\
\text { Direct }\end{array}$ & Prospectivo & $\begin{array}{l}\text { Estudar sobre a ingestão de ácidos graxos } \\
\text { (AG) ômega-3 e ômega- } 6 \text { durante a } \\
\text { gestação, a expressão de citocinas } \\
\text { inflamatórias e indução do parto }\end{array}$ & $\begin{array}{l}\text { Estudos indicaram que ômega-3 possui atividade positiva no no } \\
\text { desenvolvimento cerebral neonatal. Alto teor de AGs na dieta materna está } \\
\text { associada a duração da gestação. O estudo sugere que ômega-3 prolonga a } \\
\text { gravidez influenciando a produção de prostaglandinas envolvidas na indução } \\
\text { do parto. Há escassez de evidências sobre os efeitos da dosagem diferente } \\
\text { de ômega-3 e ômega- } 6 \text { nos perfis de citocinas no útero. }\end{array}$ \\
\hline $\begin{array}{l}\text { KARS et al } \\
\text { Reino Unido/ } \\
\quad 2016\end{array}$ & PUBMED & Meta-análise & $\begin{array}{l}\text { Avaliar os efeitos dos ácidos graxos ômega- } \\
3 \text { no início precoce }(<34 \text { semanas }) \text { e em } \\
\text { qualquer parto pré-termo }<37 \text { semanas. }\end{array}$ & $\begin{array}{l}\text { O estudo informa que ácidos graxos ômega- } 3 \text { são eficazes na prevenção de } \\
\text { partos prematuros. A intervenção é simples e facilmente disponível e tem o } \\
\text { potencial de influenciar estratégias baseadas na população na prevenção do } \\
\text { nascimento prematuro. }\end{array}$ \\
\hline $\begin{array}{l}\text { JAMILIAN et al } \\
\text { Irã/ } 2016\end{array}$ & PUBMED & Experimental & $\begin{array}{l}\text { Determinar os efeitos dos ácidos graxos } \\
\text { ômega } 3 \text { e co-suplementação com vitamina } \\
\text { E sobre biomarcadores de estresse } \\
\text { oxidativo, inflamação e resultado da } \\
\text { gravidez em mulheres com Diabetes } \\
\text { Gestacional (DG) }\end{array}$ & $\begin{array}{l}\text { O estudo foi realizado com } 60 \text { pacientes com DG sem ingestão de } \\
\text { hipoglicemiantes orais, selecionado aleatoriamente para ingestão de } 1.000 \\
\text { mg de ácidos graxos ômega-3 a partir de óleo de linhaça mais } 400 \text { Ul de } \\
\text { suplementos de vitamina } E(n=30) \text { ou placebo }(n=30) \text { por } 6 \text { semanas. Os } \\
\text { resultados mostraram que ácidos graxos ômega- } 3 \text { e a suplementação com } \\
\text { vitamina } E \text {, comparados ao placebo, resultaram em um aumento significativo } \\
\text { na capacidade antioxidante total, e menor incidências de hiperbilirrubinemia } \\
\text { em recém-nascidos }\end{array}$ \\
\hline $\begin{array}{l}\text { WARSTEDT et al } \\
\text { Suécia/ } 2016\end{array}$ & $\mathrm{NCBI}$ & Experimental & $\begin{array}{l}\text { Avaliar os ácidos graxos poliinsaturados de } \\
\text { cadeia longa ômega-3 (LCPUFA) no leite } \\
\text { materno em relação à suplementação com } \\
\text { ômega-3 e o desenvolvimento de doença } \\
\text { alérgica em seus bebês. }\end{array}$ & $\begin{array}{l}\text { O estudo foi realizado com } 95 \text { mulheres grávidas com risco alérgico para o } \\
\text { bebê, suplementadas com } 2,6 \mathrm{~g} / \text { dia de ômega- } 3 \text { LCPUFA ou placebo de } \\
2,7 \mathrm{~g} / \text { dia de óleo de soja, entre a } 25^{\mathrm{a}} \text { semana gestacional até } 03 \text { meses de } \\
\text { lactação. Os resultados apresentaram no leite altos níveis de LCPUFA- } \\
\text { ômega- } 3 \text {, em relação a mães que receberam suplementos de ômega-3 foram } \\
\text { relacionados a menos alergias em seus filhos }\end{array}$ \\
\hline $\begin{array}{l}\text { MEHER et al } \\
\text { Índia /2016 }\end{array}$ & PUBMED & Transversal & $\begin{array}{l}\text { Examinar o papel do perfil de ácidos graxos } \\
\text { ômega-3 e ômega- } 6 \text { ao longo da gestação } \\
\text { no crescimento fetal através de amostras de } \\
\text { sangue. }\end{array}$ & 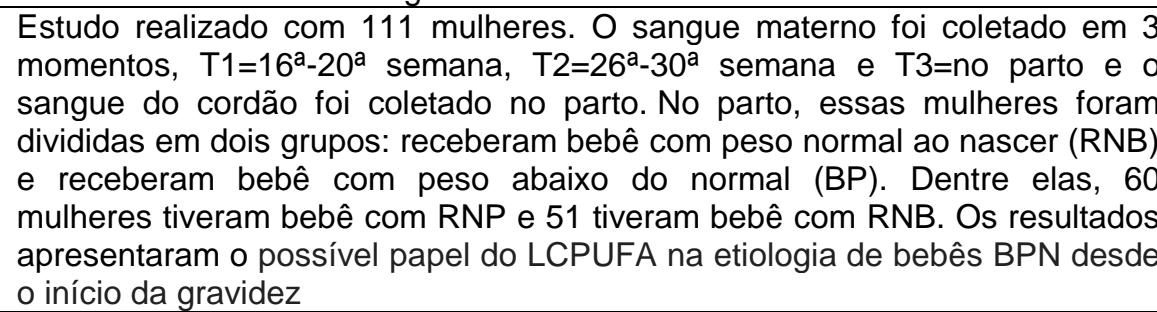 \\
\hline
\end{tabular}

REAS/EJCH | Vol. 11 (1) | e218 | DOI: https://doi.org/10.25248/reas.e218.2019 Página 6 de 10 


\begin{tabular}{|c|c|c|c|c|}
\hline $\begin{array}{l}\text { MAGALHÃES } \\
\text { Brasil/ } 2017\end{array}$ & PUBMED & $\begin{array}{l}\text { Descritivo } \\
\text { transversal }\end{array}$ & $\begin{array}{l}\text { Avaliar a ingestão nutricional e a sua } \\
\text { adequação em mulheres grávidas, } \\
\text { particularmente em relação aos ácidos } \\
\text { gordos polinsaturados ómega } 3 .\end{array}$ & $\begin{array}{l}\text { Estudo realizado com } 92 \text { mulheres com tempo de gestação entre } 32 \text { e } 40 \\
\text { semanas, onde foram coletados dados sociodemográficos e antropométricos, } \\
\text { avaliando a prevalência da inadequação da ingestão, comparando com } \\
\text { valores de referência de DRI. Os resultados sugerem ingestão inadequada de } \\
\text { ômega-3, inferior às recomendações de ácido alfa-linolénico, ácido } \\
\text { eicosapentaenóico e ácido docosahexaenóico, bem como de outros } \\
\text { micronutrientes }\end{array}$ \\
\hline $\begin{array}{l}\text { VASCONCELOS } \\
\text { et al } \\
\text { Brasil/ } 2017\end{array}$ & Scielo & Coorte & $\begin{array}{l}\text { Avaliar a ingestão de ácidos graxos alfa- } \\
\text { linolênicos em gestantes e fatores } \\
\text { associados a ingestão. }\end{array}$ & $\begin{array}{l}\text { O estudo mostrou que um terço das dietas apresentavam ingestão de ácido } \\
\text { alfa-linolênico inferior à recomendada diariamente, sendo as gestantes com } \\
\text { sobrepeso propensão duas vezes mais de ingeri-los } \\
\text { inadequadamente. Gestantes socioeconomicamente desfavorecidas } \\
\text { apresentam maiores riscos de ingestão inadequada. }\end{array}$ \\
\hline $\begin{array}{l}\text { MEREY et al } \\
\text { Brasil/ } 2017\end{array}$ & Scielo & $\begin{array}{l}\text { Experimental } \\
\text { randomizado }\end{array}$ & 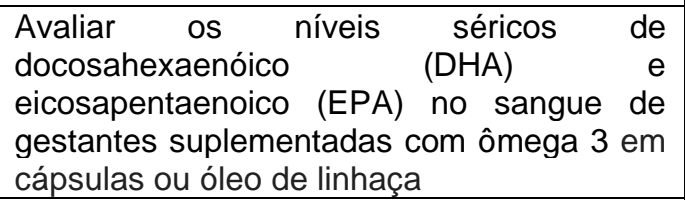 & $\begin{array}{l}\text { O estudo analisou níveis de ácidos graxos em amostras de sangue materno } \\
\text { nos períodos antes e após a suplementação e analisada diferenças entre os } \\
\text { conteúdos totais de cada grupo. Quando comparados entre si, com óleo de } \\
\text { linhaça, apresentaram-se maiores conteúdos de AA e DHA, indicando que a } \\
\text { suplementação favoreceu a formação de EPA. }\end{array}$ \\
\hline $\begin{array}{l}\text { WADHWANI } \\
\quad \text { et al } \\
\text { Índia/ } 2018\end{array}$ & $\begin{array}{l}\text { Science } \\
\text { Direct }\end{array}$ & $\begin{array}{l}\text { Prospectiva } \\
\text { longitudinal }\end{array}$ & $\begin{array}{l}\text { Investigar o papel de ácidos graxos poli- } \\
\text { insaturados de cadeia longa (LCPUFA) } \\
\text { durante a gravidez associação com } \\
\text { resultado adverso da gravidez. }\end{array}$ & $\begin{array}{l}\text { Os LCPUFA desempenham atual no crescimento e desenvolvimento do feto, } \\
\text { influenciados pelo nível e proporção de ácidos graxos ômega-3 e ômega-6 no } \\
\text { tecido. Níveis mais baixos de DHA e AA foram relatados em } \\
\text { várias complicações da gravidez. }\end{array}$ \\
\hline $\begin{array}{l}\text { MOURA E } \\
\text { AZEVEDO } \\
\text { Brasil/ } 2018\end{array}$ & Scielo & $\begin{array}{l}\text { Qualitativa } \\
\text { descritiva }\end{array}$ & $\begin{array}{l}\text { Apresenar informações relacionadas à } \\
\text { alimentação de gestantes e verificar } \\
\text { possíveis riscos de uma alimentação } \\
\text { inadequada para gestantes }\end{array}$ & $\begin{array}{l}\text { O estudo indica que o alimento ingerido pela mãe pode trazer prejuízos ou } \\
\text { benefícios ao feto. A alimentação deve ser balanceada e adequada, } \\
\text { contemplando cada período gestacional, suprindo a necessidade nutricional } \\
\text { aumentada durante a gravidez. }\end{array}$ \\
\hline $\begin{array}{c}\text { Ll et al } \\
\text { China/ } 2018\end{array}$ & $\begin{array}{l}\text { Science } \\
\text { Direct }\end{array}$ & Meta-análise & $\begin{array}{l}\text { Verificar efeitos da suplementação de } \\
\text { ácidos graxos ômega-3 durante a gestação } \\
\text { frente ao peso corporal, comprimento } \\
\text { corporal, índice de massa corporal (IMC), } \\
\text { circunferência da cintura e espessura de } \\
\text { prega cutânea da prole. }\end{array}$ & $\begin{array}{l}\text { Estudos indicaram que a suplementação de ácidos graxos ômega-3 materna } \\
\text { pode aumentar o peso da prole ao nascer, assim como a circunferência da } \\
\text { cintura no período pós-natal. No entanto, não parece influenciar o } \\
\text { comprimento do nascimento, o peso/comprimento pós-natal, o IMC, o } \\
\text { somatório das dobras cutâneas, a massa gorda e o percentual de gordura } \\
\text { corporal no período pós-natal. }\end{array}$ \\
\hline $\begin{array}{l}\text { LEWIS et al. } \\
\text { Reino Unido/ } \\
\quad 2018\end{array}$ & NCBI & $\begin{array}{l}\text { Descritivo } \\
\text { transversal }\end{array}$ & $\begin{array}{l}\text { Analisar a transferência de ácidos graxos } \\
\text { placentários e os problemas que precisam } \\
\text { ser abordados. }\end{array}$ & $\begin{array}{l}\text { O estudo relata que ácidos graxos são importantes como sinais entre a mãe, } \\
\text { a placenta e o feto, combinando a demanda fetal com a capacidade materna } \\
\text { e mediando os principais eventos, como o parto. }\end{array}$ \\
\hline $\begin{array}{l}\text { CARVALHO et al } \\
\text { Brasil/ } 2018\end{array}$ & PUBMED & $\begin{array}{l}\text { Descritivo } \\
\text { transversal }\end{array}$ & $\begin{array}{l}\text { Estudar a relevância da suplementação de } \\
\text { ômega-3 no período gestacional. }\end{array}$ & $\begin{array}{l}\text { O estudo indica que o ômega-3 tem ação sobre redução do dano oxidativo e } \\
\text { inflamação durante a gestação, prevenindo casos como pré-eclâmpsia (PE), } \\
\text { crescimento intrauterino restrito (CIUR), Diabetes gestacional (DG), depressão pós- } \\
\text { parto (DPP), dentre outros. A suplementação com ômega-3 não previne recorrência de } \\
\text { CIUR em mulheres com gravidez única, e achados demonstram redução de } 73 \% \text { na } \\
\text { morte perinatal em gestações. }\end{array}$ \\
\hline
\end{tabular}

REAS/EJCH | Vol. 11 (1) | e218 | DOI: https://doi.org/10.25248/reas.e218.2019 Página 7 de 10 


\section{DISCUSSÃO}

O tempo de gestação compreende um período de 40 semanas, com desenvolvimento embrionário e fetal. Durante esse período, as reservas minerais e nutricionais são direcionadas para a formação do bebê, acarretando em uma alteração metabólica mais expressiva na mulher gestante, em comparação com as que não se encontram em tal período. Se por ventura os níveis de vitaminas e nutrientes atingirem níveis abaixo do normal, mãe e concepto irão começar a competir por tais elementos, ocasionado prejuízos a ambos. Moura e Azevedo (2018) afirmam que a alimentação deve adequar - se ao período gestacional, para suprir as necessidades de mãe e concepto (WILLIAMS, 2011).

A ingestão de componentes nutricionais chave é de extrema importância durante a gestação, dentre os quais os ácidos graxos (AG) poli-insaturados representam uma classe necessária para o período. Os AGs são frequentemente divididos em ácido alfa-linoléico (ALA), precursor do ômega-3, que formará o ácido eicosapentaenoico (EPA) e ácido docosahexaenóico (DHA); e o ácido linoleico (LA), precursor do ômega-6, convertido a ácido araquidônico (AA) (SILVA, 2011) (WAITZBERG, 2015)

Representantes dos ômegas 3 e 6, DHA e AA, são partes constituintes dos fosfolipídios das membranas celulares, assegurando a função e fluidez da membrana, regulando as vias de sinalização celular, atuando sobre expressão gênica e servindo com substrato para a síntese de mediadores lipídicos. Com isso, uma deficiência de DHA pode estar associada com prejuízo cognitivo e ao desenvolvimento visual do concepto (MAHAN et al., 2013) (WAITZBERG, 2015).

Os AGs funcionam como mediadores anti-inflamatórios, nas gestantes alteram a produção de tromboxanos e prostaglandinas E2 e F2, envolvidas no amadurecimento do colo do útero, mecanismo proposto para evitar o trabalho de parto precoce. Também contribuem para maior peso do bebê ao nascimento, menor risco de pré-eclâmpsia, recém-nascidos pequenos para a idade gestacional e menor incidência de alergias, sendo este último, confirmado nas pesquisas de Warstedt et al (2016), em que gestante suplementadas apresentaram maiores níveis de ômega-3 no leite, e filhos com menor incidência a alergias.

Sacconi et al (2015) não compartilham a mesma conclusão, em seus estudos com revisão bibliográfica não relataram evidências suficientes para apoiar o uso rotineiro de suplementação de ômega- 3 durante a gravidez, onde a redução significativa de $73 \%$ na morte perinatal foi analisada apenas em gestações únicas, que iniciaram a suplementação com ômega $-3 \leq 20$ semanas, então afirmam que mais pesquisas são necessárias.

Merey et al (2017) afirmam a função descrita anteriormente, em que AGs do tipo ômega atuam no organismo para as funções de aprendizagem, cognição e memória. O ômega-3 possui um importante papel no tempo gestacional, atuando sobre peso, comprimento e auxiliando na formação e no crescimento dos neurônios e desenvolvimento da retina fetal (MAGALHÃES, 2017) (BEST et al., 2016) (KAR et al., 2016)

O ômega-6, por outro lado, está associado a risco de parto prematuro. Esse risco é descrito em literatura devido ao ácido araquidônico ser utilizado na síntese de prostaglandinas da série 2, o grupo responsável por iniciar do trabalho de parto, já que favorecem o amadurecimento cervical e a contração uterina (MOLTÓ-PUIGMARTí et al., 2014).

Durante o período gestacional, os AGs são enviados ao feto através da placenta, isso ocorre por não apresentarem capacidade de sintetizar gorduras, devido a ação as enzimas delta- 5 e delta- 6 dessaturases para converter ALA e LA em ômega-3 e ômega-6 ser limitada. O mecanismo pelo qual esses AGs são transportados ainda é desconhecido para a ciência. (WAITZBERG, 2015) (MAGALHÃES, 2017).

De acordo com recomendações da European Food Safety Authority (EFSA), é indispensável aumento da ingestão de DHA no período gestacional e de lactação, com a finalidade de otimizar a saúde da mãe e do concepto. A recomendação é a mesma para um indivíduo adulto, com mínimo de ingestão de $250 \mathrm{mg}$ de EPA e DHA, acrescida de 100 a 200mg de DHA ao dia, o que corresponde a duas porções de 30 a $40 \mathrm{~g} / \mathrm{dia}$ de peixes gordos de águas frias, como atum, sardinha ou salmão, principalmente no primeiro trimestre da 
gravidez, período que ocorre a formação do sistema nervoso fetal. Entretanto, estabelecer uma quantidade precisa é muito difícil, já que a capacidade da mãe de armazenar AGs e sintetizá-lo a partir de seu precursor pode variar, por diferenças naturais do organismo (PIOVESAN, 2010) (MAGALHÃES, 2017) (HAGGARTY, 2016).

Os alimentos que apresentam alto teor de AGs, encontramos linhaça (Linum usita ssimun), fonte de ômega-3, fibras e lignanas, a qual é consumida na forma de farinha ou óleo e principalmente os peixes de água fria. Uma alimentação balanceada deve apresentar uma relação entre ômega-3 e ômega- 6 na proporção de 4:1, contudo, o ritmo de vida atual muitas vezes não permite uma alimentação rica e bem combinada, baseada em alimentos criteriosamente selecionados. Sendo assim, a concentração dietética de AGs ômega-3 e ômega-6 devem estar na faixa entre 5:1 a 3:1, respectivamente. No Brasil, por se ter um alto consumo de feijão e arroz, e limitação a ingestão de peixes, a recomendação para a ingestão de AGs do tipo ômega-3 é maior (SHERRY; OLIVER; MARRIAGE, 2015) (HEATON et al., 2013).

\section{CONSIDERAÇÕES FINAIS}

Com a realização do presente trabalho de revisão, foi possível compreender a ação dos lipídios, na forma de ácidos graxos poli-insaturados das classes de ômega-3 e ômega-6, no organismo de mulheres gestantes. Os componentes lipídicos, em especial sua menor porção, os ácidos graxos, se fazem presentes em basicamente todas as formas de vida, onde desempenham funções importantes, como compondo a estrutura das membranas celulares, fazendo parte de processos metabólicos e atuando como reserva de energia. Nos seres humanos, os ácidos linoleicos, ômega- 6 e os ácidos alfa-linolênico, ômega-3, possuem a função de manter as membranas celulares, funções cerebrais e transmissão de impulsos nervosos. A ação de tais ácidos graxos é essencial no período gestacional, pois podem atuar com ação benéfica para mãe e concepto. O ômega-3 é o ácido graxo de maior contribuição no período gestacional, pois atua impedindo o parto prematuro, diminuindo o risco de pré-eclâmpsia, o risco a reações alérgicas, auxilia na formação neural e atua ainda frente ao desenvolvimento da retina. Dentre os artigos estudados, 18 artigos confirmaram tais benefícios para mãe e feto. O ômega-6, por outro lado, contribui de forma negativa durante o período gestacional, pois seu precursor, ácido araquidônico, estimula prostaglandinas do grupo 2, o que levaria a riscos de um trabalho de parto prematuro. Com relação a ingestão, não se tem um valor determinado para o período gestacional, sendo utilizado a recomendação geral para adultos, de 100 a $200 \mathrm{mg} /$ dia de DHA o valor inicial.

\section{REFERÊNCIAS}

1. AKERELE AO, CHEEMA SK. A balance of ômega-3 and ômega-6 polyunsaturated fatty acids is importante in pregnancy. Journal of nutrition \& Intermediary Metabolism. 2016; vol. 5: 23-33.

2. BARTOLOZO EAFQ, SAUER E, SANTOS MS et al. Suplementação com ácido docosahexaenóico ômega-3: influência na composição lipídica e no perfil de ácidos graxos do leite humano. Revista de nutrição. 2013; vol. 23 n. 1.

3. BEST KP, GOLD M, KENNEDY D et al. Omega-3 long-chain PUFA intake during pregnancy and allergic disease outcomes in the offspring: a systematic review and meta-analysis of observational studies and randomized controlled trials. Am J Clin Nutr. 2016;103(1):128-43

4. CAMPOS, A. B. F.; PEREIRA, R. A.; QUEIROZ et al. Ingestão de energia e de nutrientes e baixo peso ao nascer: estudo de coorte com gestantes adolescentes. Revista de Nutrição, Campinas, SP. 2013; v. 26, n. 5, p. 551-61.

5. CARVALHO SMB, SANTOS JBN, QUADROS IAAO, JUNQUEIRA ML et al. Suplementaçaõ de ômega-3 no período gestacional: aspectos relevantes. International Journal of Nutrology. 2018; 11(S 01):S24-S327.

6. ESCAMILLA-NUÑEZ MC, BARRAZA-VILLARREAL A, HERNÁNDEZ-CADENA L et al. Omega-3 fatty acid supplementation during pregnancy and respiratory symptoms in children. Chest. 2014; 146 (2): 373-382.

7. FAO. Fats and fatty acids in human nutrition. Report on expert consulation. Food Nutrition Paper 91.2010. FEREIDOONI B, JENABI E. The use of ômega 3 on pregnancy outcomes: a single-center study. J Park Med Assoc. 2014; 64 (12): 1363-5.

8. GRIGORIADIS S, BARRET J, PITTINI $R$ et al. Omega-3 supplements in pregnancy: are we too late to identify the possible benefits? Journal of obstetrics and gynaecology Canada. 2010; vol. 32, 3:209-216.

9. GOULD JF, ANDERSON AJ, YELLAND LN et al. Maternal characteristics influence response to DHA during pregnancy. Prostaglandins Leukot Essent Fatty Acids. 2016;108:5-12.

10. GUNARTE AW, MAKRIDES M, COLIINS CT. Maternal prenatal and/or pós-natal $n-3$ ling chain polyunsaturated fatty acids (LCPUFA) supplementation for preventing allergies in early childhood. The Cochrane Database os Systematic Reviews. 2015; v.7, p. CD010085.

11. HAGGARTY P, ASHTON J, JOYNSON M et al. Effect of maternal polyunsaturated fatty acid concentration on transport by the human placenta. Biol Neonate. 2016; 75(6):350-9 
12. HEATON AE, et al. Does docosahexaenoic acid supplementation in term infants enhance neuro cognitive functioning in infancy? Frontiers in Human Neuroscience. 2013; v.7, 774, p. 1-12.

13. INNIS SM. Impacto f maternal diet on human milk composition and neurological development os infants. American Journal os Clinical Nutrition. 2014; v. 99, n.3, p. 734S-741S

14. JAMILIAN M, HASHEMI DIZAJI S, BAHMANI, M et al. A randomizes controlled clinical trial investigating the effects of ômega-3 fatty acids and vitamin E co-supplementation o biomarkers of oxidative stress, inflammation and pregnancy outcomes in gestational diabetes. Can J Diabetes. 2017: 41 (2): 143-149.

15. LEWIS RM, CHILDS, CE, CALDER PC. New perspectives on placental fatty acid transfer. Prostaglandins Leukot Essent Fatty Acids. 2018 Nov;138:24-29.

16. LI G, CHEN H, ZHANG W, et al. Effects of maternal ômega-3 fatty acids supplementation during preagnancy/ lactation on body composition os the offspring: A systematic review and meta-analysis. Clinical Nutrition. 2018; vol. 37, 5: 1462-1473.

17. KAR S, WONG M, ROGOZINSKA E et al. Effects of omega-3 fatty acids in prevention of early preterm delivery: a systematic review and meta-analysis of randomized studies. Eur J Obstet Gynecol Reprod Biol. 2016; 198:40-6

18. KOLETZKO, B.; CETIN, I.; BRENNA, J. T.; Perinatal Lipid Intake Working Group; Child Health Foundation; Diabetic Pregnancy Study Group; European Association of Perinatal Medicine; European Association of Perinatal Medicine; European Society for Clinical Nutrition and Metabolism; European Society for Paediatric Gastroenterology; Hepatology and Nutrition; Committee on Nutrition; International Federation of Placenta Associations; International Society for the Study of Fatty Acids and Lipids. Dietary fat intakes for pregnant and lactating women. The British Journal of Nutrition. 2007; v.98, n.5, p.873-877.

19. MAGALHÃES D. Avaliação da ingestão de ácidos gordos polinsaturados ômega 3 numa amostra de grávidas. Dissertação (Mestrado em Nutrição Clínica) - Faculdade de Ciências da Nutrição e Alimentação. Universidade do Porto. Porto, $2017 ; 45$ p.

20. MAHAN KL, ESCOTT-STUMP S, RAYMOND J. Alimentos, Nutrição e Dietoterapia. 2013. 13ª ed.

21. MEHER A, RANDHIR K, MEHENDALE $S$ et al. Maternal fatty acids and their association with birth outcome: a prospective study. PloS One. 2016. 27; 11(1).

22. MENDES KDS, SILVEIRA RCCP, GALVÃO CM. Revisão integrativa: método de pesquisa para a incorporação de evidências na saúde e na enfermagem. Texto contexto enferm., 2008; 17(4): 758-764

23. MOLTÓ-PUIGMARTI C, VAN DONGEN MC, DAGNELIE PC et al. Maternal but not fetal FADS gene variants modify the association between maternal long-chain PUFA intake in pregnancy and birth weight. The Journal of Nutrition. 2014; v.144, n.9, p.1430-1437.

24. MOURA AR, AZEVEDO FHC. Evidências científicas sobre a alimentação de gestantes. Revista Saúde em Foco, Teresina. 2018 ; v. 5, n. 1, art. 5, p. 78-90.

25. OLIVEIRA, J. M; LUZIA, L. A; RONDÓ, P. H. C. Ácidos Graxos Poli-insaturados Ômega-3: saúde cardiovascular e sustentabilidade ambiental. Segurança Alimentar e Nutricional. 2012; v. 19, n. 1, p. 89-96, Campinas.

26. PIOVESAN CH. Efeito da Modificação do Estilo de Vida Sobre a Qualidade da Dieta em Indivíduos com Síndrome Metabólica. Dissertação (Mestrado em Medicina e Ciências da Saúde). Pontifícia Universidade Católica do Rio Grande do Sul, Porto Alegre, 2010.

27. SACCONE G, SACCONE I, BERGHELLA V. Omega-3 long-chain polyunsaturated fatty acids and fish oil supplementation durin pregnancy: which evidence? J. Matern. Fetal Neonatal Med. 2016; 29 (15): 2389-97.

28. SILVA JAP. Efeito da suplementação de óleo de peixe em marcadores da resposta inflamatória e do estado nutricional em indivíduos adultos com câncer colo-retal. Dissertação (Mestrado em Nutrição). Universidade Federal de Santa Catarina. Florianópolis, 2011.

29. SIMOPOULOS AP. Omega-3 fatty acids in inflammatory and autoimmune diseases. Journal of the American College of Nutri on. 2002; v. 21, n. 6, p. 495-505.

30. SHERRY CL, OLIVER JS, MARRIAGE BJ. Docosahexaenoic acid supplementation in lactating women increases breast milk and plasma docosahexaenoic acid concentrations and alters infant omega 6:3 fatty acid. Prostaglandins Leukot Essent Fa y Acids. 2015; v. 95, p. 63-9.

31. VASCONCELOS LG, GOMES, CB, MALTA MB et al. Ingestão insuficiente de ácido graxo alfa-linolênico (18: 3n-3) durante a gestação e os fatores associados. Revista de Nutrição. 2017; vol. 30, n. 4.

32. WADHWANI N, PATIL V, JOSHI S. Maternal long chain polyunsaturated fatty acid status and pregnancy complications. 2018; vol. 136: 143-152.

33. WAITZBERG DL. Ômega-3: o que existe de concreto? 2015; Universidade de São Paulo.

34. WARSTEDT K, FUHUHJELM C, FÄLTH-MAGNUSSON K et al. High levels of ômega-3 fatty acids in milk from ômega-3 fatty acidsupplemented mothers are related to less immuniglobulin E-associated disease in infancy. Acta Paediatr. $2016 ; 105$ (11): $1337-$ 1347.

35. WILLIAMS PJ, MISTRY HD. The Importance of Antioxidant Micronutrients in Pregnancy. Hindawi Publishing Corporation. Oxidative Medicine and Cellular Longevity, 2011; 1-12, jun.

36. YU YM, CHAN YH, CALDER PC et al. Maternal PUFA status and offsping allerguc diseases up to the age of 18 months. Br J Nutri. 2015; 113 (6): 975-83.

37. ZHAO, J. P.; LEVY, E.; FRASER, W. D et al. Circulating docosahexaenoic acid levels are associated with fetal insulin sensitivity. PLoS One. 2014; v.9, n.1, p.e85054. 